\title{
Stereoscopic PIV measurement of boundary layer affected by DBD actuator
}

\author{
Pavel Procházka ${ }^{1, a}$, Václav Uruba $^{1}$ \\ ${ }^{1}$ Institute of Thermomechanics, Czech Academy of Sciences, Czech Republic
}

\begin{abstract}
The effect of ionic wind generated by plasma actuator on developed boundary layer inside a narrow channel was investigated recently. Since the main investigated plane was parallel to the channel axis, the description of flow field was not evaluated credibly. This paper is dealing with cross-section planes downstream the actuator measured via 3D time-resolved PIV. The actuator position is in spanwise or in streamwise orientation so that ionic wind is blown in the same direction as the main flow or in opposite direction or perpendicularly. The interaction between boundary layer and ionic wind is evaluated for three different velocities of main flow and several parameters of plasma actuation (steady and unsteady regime, frequency etc.). Statistical properties of the flow are shown as well as dynamical behaviour of arising longitudinal vortices are discussed via phase-locked measurement and decomposition method.
\end{abstract}

\section{Introduction}

This article is devoted to investigation of the influence of plasma DBD (dielectric barrier discharge) actuator on zero-pressure gradient boundary layer (BL) developed inside a narrow channel in order to use it for active flow control of flow separation over GG (GlaubertGoldschmiedt) body-HUMP [1]. Plasma actuators belong to the active flow control device group and have many advantages in comparison with others as synthetic jets. For example, they have no moving mechanical parts, they can be easily fastened to the surface and they have very short response time [2]. Conversely, they have quite low efficiency of energy conversion. Generally, plasma DBD actuators which are powered by alternating current, are used to control in steady regime when instantaneous ionic wind (wall-jet like flow) is produced $[3,4,5]$. There is also an unsteady regime [6] which occurs after that rectangular modulation is applied. Then vortex train is presented inside ionic wind, whereas the vortex parameter is given by electrical parameter as modulation frequency and duty cycle. The plasma actuator unsteady regime is more effective and is in the interest of research to investigate the behaviour which is to be used for effective control of boundary layer over GG body.

Previously, the effect of DBD actuator on BL was observed in narrow channel when the ionic wind has been oriented in the parallel direction as the channel floweither same or opposite direction. Further, the effect of perpendicularly oriented ionic wind on $\mathrm{BL}$ was observed also, whereas the measurement plane was in the middle of channel width and perpendicular to the bottom side
$[7,8]$. The biggest effect on BL was observed for lower velocities $(5$ or $10 \mathrm{~m} / \mathrm{s})$ and for steady actuation where velocity gain was up to $10 \%$ in normalized velocity profile. The effect of modulation frequency was obvious but only for spanwise configuration and in streamwise configuration was no effect at all which is not in agreement with previously gained results and this effect should be investigated in cross-section plane also. That is why stereoscopic TR PIV measurement was suggested in this paper for cross-section plane perpendicular to the channel flow.

This article will report about flow field behind plasma DBD actuator in base case (no actuation), in steady and in unsteady case. Time-resolved PIV was suggested as main experimental method and no numerical simulation are not incorporated. The effect of ionic wind oriented in the same/opposite/perpendicular direction to $\mathrm{BL}$ is studied and huge longitudinal vortical structure is observed in two distinct planes. There are two approaches how to describe the flow. The first one is statistical approach where mean flow field is computed from long ensemble of measurement. Phase-lock measurement was used to describe several individual phases of vortex motion. Dynamical approach utilized fast acquisition frequency $(500 \mathrm{~Hz})$ because fast dynamical processes are under investigation via analysis algorithm (POD).

\section{Description of experiment}

Plasma DBD actuator is used in simple configuration (two electrodes) in wire configuration. This means that it

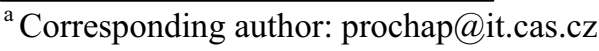


consists of two electrodes and a dielectric layer between them. Upper electrode is powered by high-voltage highfrequency waveform and is actually very thin wire (30 $\mu \mathrm{m})$ used commonly in HWA. Lower electrode is made from gold glaze, its thickness is only a few $\mu \mathrm{m}$ and the electrode width is $18 \mathrm{~mm}$. The electrode gap is $2 \mathrm{~mm}$. The dielectric layer is made from silica glass with thickness of only $1 \mathrm{~mm}$. Silica glass has good dielectric strength and it enables to use sufficient value of voltage. Lower electrode is grounded and is encapsulated to retain the plasma discharge only at the top side of actuator resulting in better efficiency.

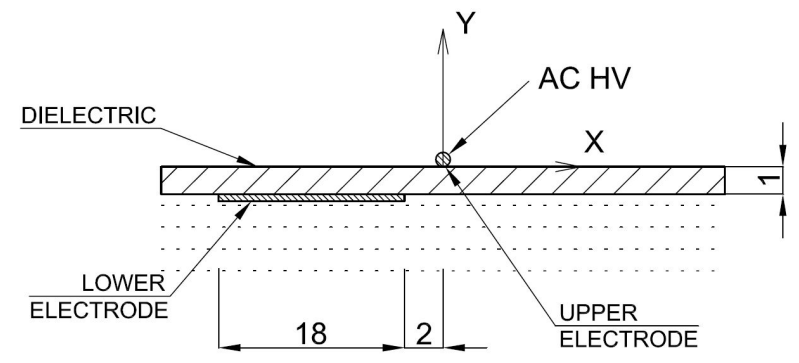

Figure 1. Plasma DBD actuator - wire type

The actuator is used in spanwise (figure 1) orientation as well as in streamwise [8] orientation. The actuator anchor points had to be replaced to the bottom part to not affect developed BL in both orientation mainly in streamwise one. Now, all anchor points and preloading mechanism of wire electrode are placed below. The platform dimension of plasma actuator is $150 \times 100 \mathrm{~mm}$ to be easily inserted inside a channel. The position of wire electrode in spanwise orientation is in the middle of actuator length $(150 \mathrm{~mm})$ which means that wire electrode is oriented across the channel width. On the other hand, the position of electrode in streamwise orientation is not in the middle of the width but is shifted to one side $(17 \mathrm{~mm})$. The right-hand side coordinate system is oriented so that $\mathrm{x}$ is in the direction of channel flow and $y$ is perpendicular to the bottom side. The power source parameter were introduced in detail in $[7,8]$ and did not undergo any alternatives. It can generate alternating voltage waveform with maximal value around $12 \mathrm{kV}$. The frequency range is adjustable but it was used fixed value of $16 \mathrm{kHz}$. The main feature of this power source is a shut-down function which enables to use rectangular modulation of voltage signal. Then another two parameters is needed to take into account modulation frequency and duty cycle (DC). This regime was called as unstable regime of actuation. When the signal is not modulated (steady regime), the generated ionic wind is observed as wall-jet like flow, parallel to the actuator surface oriented from powered electrode to the grounded one and maximal value gains up to one millimeter above the surface. The unstable regime is characterized by alternating existence of plasma discharge resulting in existence of series of vortices inside ionic wind, so called vortex train. This regime is mainly under investigation from dynamical point of view.
The boundary layer was developed inside a rectangular perspex channel connected to the blow-down wind tunnel [9]. The cross-section dimension was $100 \mathrm{x}$ $250 \mathrm{~mm}$. The total length was $3000 \mathrm{~mm}$. The actuator was placed inside channel so that it created a part of bottom side and the distance from inlet edge to the actuator center was $2275 \mathrm{~mm}$. The spanwise orientation generated ionic wind in the same/opposite orientation in comparison with channel flow. The streamwise orientation generates ionic wind in perpendicular direction.

The boundary layer was developed without any turbulizators under channel flow velocities 5, 10 and 20 $\mathrm{m} / \mathrm{s}$. Corresponding Re number are given in table 1 . The boundary layer thickness was determined from PIV 2D data in place where actuator is located without actuation and were 54, 51 and $41 \mathrm{~mm}$, respectively.

Table 1. Reynolds number for rectangular channel.

\begin{tabular}{|c|c|c|}
\hline & $\mathbf{R e}_{\mathbf{x}}$ & $\mathbf{R e}_{\boldsymbol{\delta}}$ \\
\hline $5 \mathrm{~m} \cdot \mathrm{s}^{-1}$ & $750 \cdot 10^{3}$ & $18 \cdot 10^{3}$ \\
\hline $10 \mathrm{~m} \cdot \mathrm{s}^{-1}$ & $1500 \cdot 10^{3}$ & $34 \cdot 10^{3}$ \\
\hline $20 \mathrm{~m} \cdot \mathrm{s}^{-1}$ & $3000 \cdot 10^{3}$ & $55 \cdot 10^{3}$ \\
\hline
\end{tabular}

This experiment was carried out for three basic regimes. The first one, when actuator is off, is used as base case. The steady regime is characterized by continuous ionic wind. Since unsteady regime enables to tune actuator by changing two parameters, it has become as the most researched case. The modulation frequency range from $10 \mathrm{~Hz}$ to $50 \mathrm{~Hz}$ and duty cycle was set to $30 \%$.

Stereoscopic TR-PIV was used as a measurement technique in cross-section plane, which means that all three velocity components are specified. Laser New Wave Pegasus Nd:YLF with double head and cylindrical optics was used. The wavelength is $527 \mathrm{~nm}$, maximal operation frequency is $10 \mathrm{kHz}$ and energy of one pulse is $10 \mathrm{~mJ}$ (for $1 \mathrm{kHz}$ ). We used two identical CCD cameras NanoSense MkIII with resolution of $1280 \times 1024$ and maximal sampling frequency $512 \mathrm{~Hz}$. Total memory of camera was 4 GB. Scheinpflug criterion was fulfilled and specific target moving in $\mathrm{x}$-direction was used to calibrate precisely all three dimensions. With respect to fulfill all specific features [10], the precision of this method is within 1-2 \%. The software DynamicStudion ver. 3.41 was used.

The acquisition frequency differs in dependency on data treatment. The frequency was $100 \mathrm{~Hz}$ for at least 10 second for statistical data treatment and $500 \mathrm{~Hz}$ and at least 2 second for dynamical one. Phase-locked measurement was conducted for unstable case. 20 phases was taken during one period for 7.5 second resulting in averaging over 150 period for $10 \mathrm{~Hz}$ - case. However, modulation frequency of $20 \mathrm{~Hz}$ has required two times higher acquisition frequency resulting in half measurement time. For modulation frequency $50 \mathrm{~Hz}$, it 
was necessary to capture only 10 phases per period due to limited camera memory.

\section{Results}

The character of longitudinal vortex structures generated behind plasma actuator oriented in streamwise position can be clearly seen from mean flow field evaluated in two positions distanced $110 \mathrm{~mm}$ from each other. Figure 2 shows typical image of vortex oriented in left-hand side of the channel flow. There is a position of wire electrode marked as a black spot. The position of vortex core differs for first and second position. However this difference is always equal for steady and for unsteady (for 10, 20 and $50 \mathrm{~Hz}$ ) regimes. The vortex core is inclined about $5.5^{\circ}, 3.4^{\circ}$ and $2.6^{\circ}$ for channel velocities 5,10 and $20 \mathrm{~m} / \mathrm{s}$, respectively. This corresponds with previously gained results, where the effect of $20 \mathrm{~m} / \mathrm{s}$ was the smallest one.

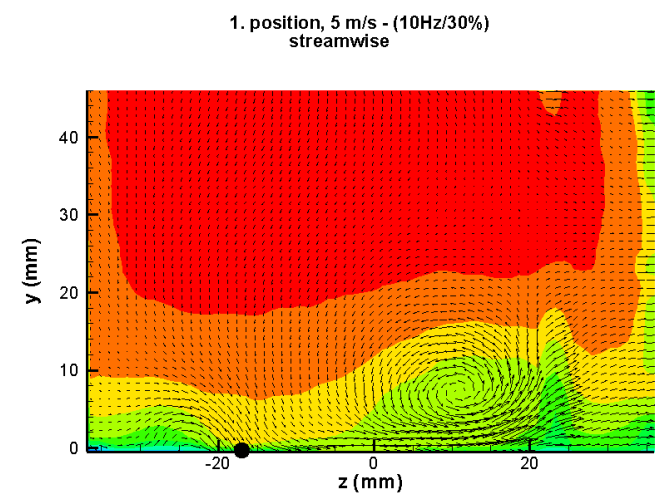

Figure 2. The cross-section of longitudinal vortex

The position of mean vortex core strongly differs in dependency on used channel velocity (see figure 2 and 3 ). When the steady regime is used, the position of vortex core is rather in the middle of the channel. If the unsteady regime of the actuator is applied, the vortex core is shifted toward the channel wall. The vortex core does not occur so far away from the centre with increasing value of modulation frequency (figure 4). It is obvious that lower value of modulation frequency has biggest effect.

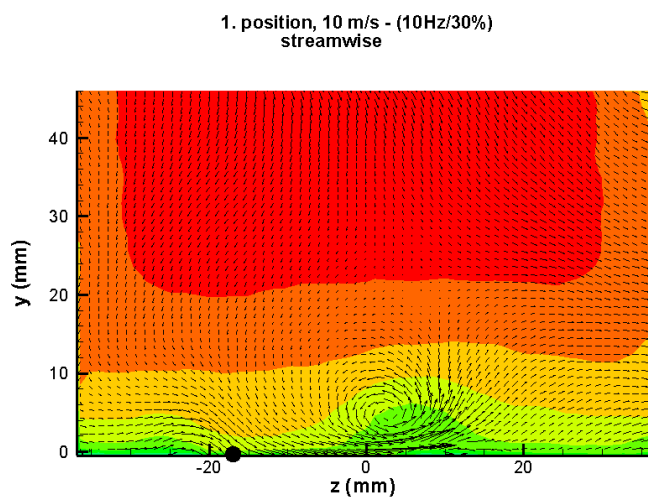

Figure 3. The cross-section of longitudinal vortex
Figures 5 and 6 show typical image of flow field generated by actuator in spanwise position. It can be seen that plasma can add momentum into boundary layer, hence the velocities are accelerated and boundary layer is more stable [7]. The flow in cross-section plane is axisymmetrical. These results perfectly correspond with results gained by 2D PIV in longitudinal plane and it shows that biggest effect on velocities has steady regime.

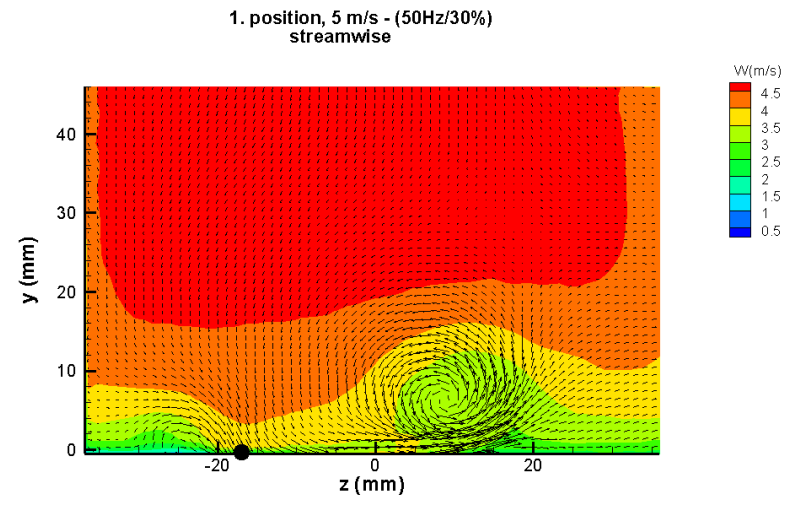

Figure 4. The cross-section of longitudinal vortex

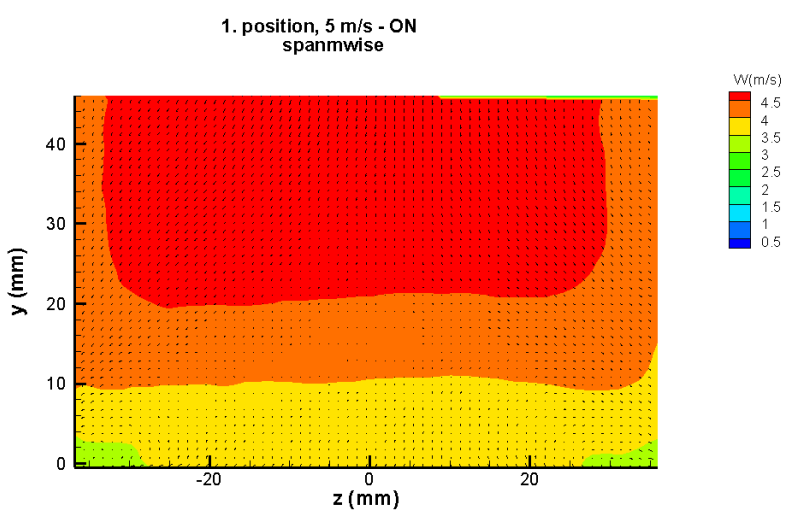

Figure 5. Boundary layer behind actuator, steady regime

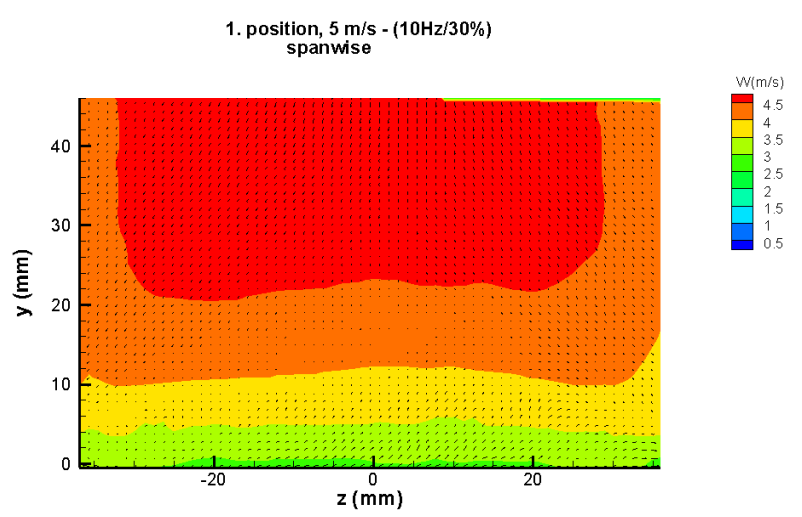

Figure 6. Boundary layer behind actuator, unsteady regime

The phase-locked measurement was done and 20 phases per period was taken for unsteady regime generated by $10 \mathrm{~Hz}$. The position of vortex core (figure $7)$ was evaluated during one period ( 0.1 second) and vortex core shifting velocity was determined as approx. 
$0.6 \mathrm{~m} / \mathrm{s}$. The vortex core was propagating all the time toward the wall until the 8 . phase, when the motion was stopped, although the plasma discharge continued until the 13. phase. Then the vortex started to fade.

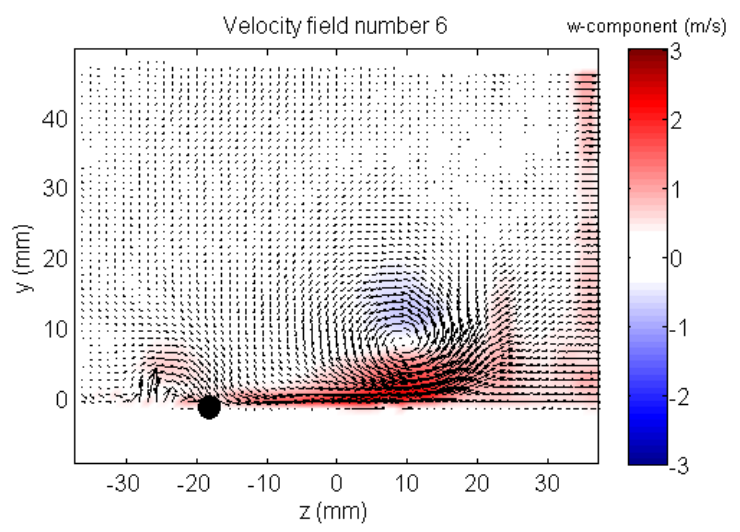

Figure 7. 6. phase of vortex core motion of one period

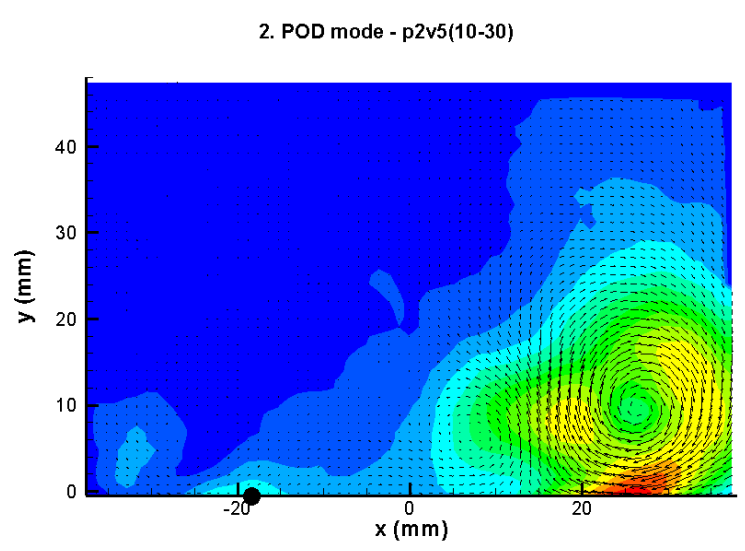

Figure 8. Second POD mode for unsteady actuation

POD analysis was applied on data taken during steady and unsteady regimes to show energy-important modes. The table 2 shows the energy distribution of first five modes. It seems that modes in second position (three right-hand side columns) are slightly more powerful. The modes with biggest energy content were found out for unsteady cases.

Table 2. Five most important modes sorted by kin. energy

\begin{tabular}{|c|c|c|c|c|c|}
\hline $\begin{array}{c}\text { p 1 } \\
\text { steady }\end{array}$ & $\begin{array}{c}\text { p 1 } \\
\text { un- } \\
\text { steady }\end{array}$ & $\begin{array}{c}\text { p 1 } \\
\text { base } \\
\text { case }\end{array}$ & $\begin{array}{c}\text { p 2 } \\
\text { steady }\end{array}$ & $\begin{array}{c}\text { p 2 } \\
\text { un- } \\
\text { steady }\end{array}$ & $\begin{array}{c}\mathrm{p} \mathrm{2} \\
\text { base } \\
\text { case }\end{array}$ \\
\hline 4,48 & 12,86 & 4,72 & 5,44 & 13,97 & 5,1 \\
\hline 3,73 & 5,38 & 3,94 & 4,85 & 7,74 & 4,17 \\
\hline 2,64 & 3,01 & 2,39 & 2,83 & 2,71 & 2,54 \\
\hline 2,03 & 2,59 & 2,29 & 2,32 & 2,21 & 2,29 \\
\hline 1,86 & 2,27 & 1,97 & 2,18 & 2,16 & 2,13 \\
\hline
\end{tabular}

\section{Conclusion}

The cross-section plane of rectangular channel was investigated experimentally by stereoscopic PIV measurement. The developed boundary layer was influenced by plasma DBD actuator which was mounted in spanwise and in streamwise orientation. The flow field was evaluated from statistically gained data, from phaselocked measured data and from dynamical analysis. The velocity distribution demonstrated better properties (e.g. stability etc.) for spanwise orientation when ionic wind was blowed in the same direction as channel flows. The streamwise orientation is characterized by perpendicularly blowed ionic wind resulting in long inclined vortical structure whose behaviour is strongly dynamical, especially in horizontal sense.

The POD analysis revealed that the most powerful POD modes were found for unsteady actuation as the interaction between boundary layer and the vortex train inside ionic wind generates biggest structures. However, to assign distinct frequency to particular mode, more advanced analysis is required e.g. OPD. To reconstruct the flow field more precisely, several additional crosssection planes should be measured as well as planes parallel to channel bottom side.

\section{Acknowledgement}

The authors gratefully acknowledge financial support of the Grant Agency of the Czech Republic, No. GP1425354P.

\section{References}

1. J. W. Naughton, S. A. Viken, D. Greenblatt, AIAA Journal 44, 1255-1265 (2006)

2. E. Moreau, Journal of Physics D: Applied Physics 40, 605-636 (2007)

3. Y. Li, X. Zhang, X. Huang, Exp. Fluids 49, 367377 (2010)

4. N. Bénard, J. Jolibois, E. Moreau, R. Sosa, G. Artana, G. Touchard, Thin Solid Films 516, 6660-6667 (2008)

5. G. I. Font, AIAA Journal 44, 1572-1578 (2006)

6. N. Benard, N. Balcon, G. Touchard, E. Moreau, Exp. Fluids 45, 333-355 (2008)

7. P. Procházka, V. Uruba, Journal of Physics: Conference Series 1 (2014)

8. P. Procházka, V. Uruba, Conference on Modelling Fluid Flow, Budapest (2015)

9. P. Jonáš, O. Mazur, V. Uruba, IV. Watermanagement conference, Praha (2004)

10. M. Raffel, C. Willert, S. Wereley, J. Kompenhans, Particle image velocimetry, Springer-Verlag (2007) 